\title{
Further Comments on the Calculation of Position Line Data with a Computer Calculator
}

\author{
John A. Read
}

Mr. PODMore's article ${ }^{1}$ will certainly cause several people to write to you, as he is not suggesting the best use of the HP-35. One advantage of the HewlettPackard calculators is that one does not need to convert degrees and minutes to degrees and fractions of a degree before beginning the problem. Also one should use the cosine law again to find the azimuth. The usefulness of the calculator is that it can find with ease, both the calculated zenith distance and azimuth (no more tables!).

Beginning with the same basic spherical cosine formula as Mr. Podmore, only replacing the Local Hour Angle by the angle at $Z$ (which gives Azimuth) we have:

$$
\cos \mathrm{Z}=(\cos \mathrm{PX}-\cos \mathrm{PZ} \cos \mathrm{XZ}) /(\sin \mathrm{PZ} \sin \mathrm{XZ})
$$

where as before $\mathrm{ZX}=$ Zenith distance

$$
\begin{aligned}
& \mathrm{PZ}=\text { Co-lat } \\
& \mathrm{PX}=\text { Polar distance }
\end{aligned}
$$

Again replacing PZ by 90-Lat and PX by $90-\mathrm{Dec}$ (or $90+\mathrm{Dec}$ ) we find that, when Lat and Dec have the same name;

$$
\cos Z=(\sin (\mathrm{Dec})-\sin (\text { Lat }) \cos \mathrm{XZ}) / \cos (\text { Lat }) \sin \mathrm{XZ}
$$

and when Lat and Dec have different names:

$$
\cos Z=(-\sin (\mathrm{Dec})-\sin (\text { Lat }) \cos \mathrm{XZ}) / \cos (\text { Lat }) \sin \mathrm{XZ} .
$$

Let us finish the problem begun in the last article in which

$$
\begin{aligned}
& \text { DR Lat }=53^{\circ} 10^{\prime} \mathrm{N} \\
& \text { Dec }=15^{\circ} 0.3^{\prime} \mathrm{N} \\
& \mathrm{XZ}=39^{\circ} 7.6^{\prime}
\end{aligned}
$$

Substituting in the above formula (Lat and Dec Same Name) we get : $\cos Z=\left\{\sin \left(15^{\circ} 0.3^{\prime}\right)-\sin \left(53^{\circ} 10^{\prime}\right) \cos \left(39^{\circ} 7.6^{\prime}\right)\right\} /\left\{\cos \left(53^{\circ} 10^{\prime}\right) \sin \left(39^{\circ} 7.6^{\prime}\right)\right\}$ 
The necessary sequence of operations on the calculator is shown below.

Press

See

1) .3 ENTER

0.3000

2) $60 \div$

0.0050

3) $15+$

$15.005^{\circ}$

4) SIN

0.2589

5) 10 ENTER

10.0000

6) $60 \div$

7) $53+$

0.1667

$53 \cdot 1667$

8) SIN

0.8004

9) 7.6 ENTER

$7 \cdot 6000$

10) $60 \div$

0.1267

11) $39+$

$39 \cdot 1267$

12) $\mathrm{COS}$

$0.775^{8}$

I3) $\mathrm{X}$

14) -

15) I० ENTER

0.6209

$-0.3620$

10.0000

0.1667

16) $60 \div$

$53 \cdot 1667$

17) $53+$

18) $\mathrm{COS}$

0.5995

19) 7.6 ENTER

7.6000

20) $60 \div$

0.1267

21) $39+$

$39 \cdot 1267$

22) SIN

0.6310

23) $\mathrm{X}$

0.3783

24) $\div$

$-0.9569$

25) $\mathrm{COS}^{-1}$

$163 \cdot 1174$

min to degrees

min to degrees

min to degrees

product of sine and cosine

value of top line

min to degrees

min to degrees

The Azimuth is $163^{\circ} 1^{\circ}$ or $196.9^{\circ}$.

Although only four decimal places are shown the calculator computes with io significant figures. The HP model used above is the HP-2 1 which is one half the cost of the HP-35.

One final comment, the HP- 35 is also far less expensive than the HP-65 and again is more useful for a navigator, especially with its built in 'stop-watch'. I find it amusing that the revolution in calculators is occurring so fast that Mr. Podmore's article refers to out-dated equipment-and next month this article will possibly be out of date too!

\section{REFERENCE}

1 Podmore, H. L. (1975). The calculation of position line data with a computer calculator. This Journal, 28, 101 . 